\title{
Steps that count: Pedometer-measured physical activity, self- reported physical activity and current physical guidelines - how do they relate?
}

\author{
J D Pillay, ${ }^{1,2} \mathrm{PhD}$; T L Kolbe-Alexander, ${ }^{1} \mathrm{PhD} ;$ K I Proper, ${ }^{3} \mathrm{PhD} ;$ S A Tomaz, ${ }^{1}$ MPhil; W van Mechelen, ${ }^{3} \mathrm{PhD} ; \mathrm{E} \mathrm{V} \mathrm{Lambert,}{ }^{1} \mathrm{PhD}$ \\ ${ }^{1}$ UCT/MRC Exercise Science and Sports Medicine Research Unit, Faculty of Health Sciences, University of Cape Town, South Africa \\ ${ }^{2}$ Department of Basic Medical Sciences, Faculty of Health Sciences, Durban University of Technology, South Africa \\ ${ }^{3}$ Department of Public and Occupational Health, EMGO+ Institute for Health and Care Research, VU University Medical Centre, Amsterdam, The Netherlands
}

Corresponding author: J D Pillay (pillayjd@dut.ac.za)

Background. The association between self-perceived and actual physical activity, with particular reference to physical activity guidelines, may be an important factor in determining the extent of uptake of and compliance with physical activity.

Objectives. To examine the association between self-perceived and actual physical activity in relation to physical activity guidelines, with reference to volume, intensity and duration of steps/day, and to establish the level of agreement between pedometer-measured and selfreported ambulatory physical activity, in relation to current guidelines.

Methods. A convenience sample of adults ( $N=312$; mean (standard deviation) age 37 (9) years), wore a pedometer (minimum 3 consecutive days) and completed a questionnaire that included information on physical activity patterns. Analyses of covariance, adjusted for age and gender, compared volume- and intensity-based steps according to meeting/not meeting guidelines (self-reported). The extent of agreement between self-reported and pedometer-measured physical activity was also determined.

Results. Average (SD) steps/day were 6574 (3 541). Of a total of 312 participants's self-reported data, those meeting guidelines ( $n=63$ ) accumulated significantly more steps/day than those not meeting guidelines (8 753 (4 251) v. 6022 (3 114) total steps/day and 1772 (2 020) v. 421 (1 140) aerobic steps/day, respectively; $p<0.0001)$. More than half of the group who self-reported meeting the guidelines did not meet guidelines as per pedometer data.

Conclusion. The use of pedometers as an alternative and/or adjunct to self-reported measures is an area for consideration. Steps/day recommendations that consider intensity-based steps may provide significant effects in improving fitness and health.

S Afr J SM 2014;26(3):77-81. DOI:10.7196/SAJSM.534

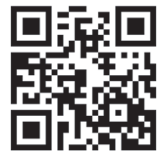

Studies on physical activity behaviours and their association with morbidity and mortality rates have largely been measured through self-reported (indirect) measures of physical activity using interviews, surveys, questionnaires and diaries/logs. ${ }^{[1,2]}$ These measures are frequently used owing to their practicality, including low cost, low participant burden and general acceptance. ${ }^{[2]}$ Although self-reported data can provide useful insights into the physical activity levels of populations or subgroups, these data have the tendency to over- or underestimate true physical activity energy expenditure and rates of inactivity. ${ }^{[1]}$ Furthermore, issues around recall and differential interpretations of terms present an evident bias. ${ }^{[3]}$ Self-reported measures are also usually unable to capture the absolute level of physical activity ${ }^{[4]}$ and are therefore typically considered subjective.

Direct measures have the potential to provide more precise estimates of energy expenditure and may reduce/eliminate many of the limitations of recall-and-response bias evident in indirect measures. ${ }^{[5]}$ Few studies ${ }^{[6,7]}$ have attempted to measure the level of agreement between self-reported measures and steps/day data as a direct/objective measure of ambulatory physical activity. ${ }^{[8-10]}$
Furthermore, such studies have been accelerometer based. This is primarily due to the fact that pedometers traditionally presented volume-based information, with little or no reference to intensity or duration of intensity-based ambulatory physical activity or its relationship to current physical activity guidelines. With more recent literature providing intensity-based step recommendations (such as 3000 steps in 30 minutes), ${ }^{[11-13]}$ the need to incorporate elements of current guidelines, including volume, intensity, duration and frequency of physical activity, into pedometer-based messages has therefore become an area of increased research. Such research continues to strengthen so as to ensure congruency between pedometer-based recommendations and physical activity guidelines.

Recent advancements in pedometry create the opportunity for its use in providing more detailed information on physical activity patterns, rather than simply recording a tally of steps/day, even though it is limited to ambulatory physical activity. For example, the ability to provide information on intensity-based step ${ }^{[14]}$ may provide a more objective alternative, or complement, to self-reported approaches that typically rely on recall. Using pedometers for such a purpose would also be less costly than instruments such as accelerometers, used 
for providing an objective measure of ambulatory physical activity. Consequently, the opportunity arose to evaluate the relationship between pedometer-determined physical activity patterns and selfreported ambulatory physical activity, in terms of volume, intensity and duration of steps/day.

\section{Objectives}

The objectives of our study were, therefore, to compare the volume and intensity of steps/day using pedometer-measured and selfperceived (questionnaire) measures, in relation to current physical activity guidelines, and to establish the level of agreement between pedometer-measured and self-perceived ambulatory physical activity, in relation to current guidelines.

\section{Methods}

This was a cross-sectional study among employed South African adults.

\section{Participant recruitment}

A convenience sample of participants was recruited through an invitation email sent out to employees, or following the completion of a health risk screening hosted primarily at corporate organisations. The corporate organisations mainly comprised health-insured, whitecollar workers. The physical activity levels required by and performed in most of these jobs were low in general.

\section{Pedometer wear}

Participants were requested to wear a blinded (Omron HJ 720 ITC) pedometer, attached to the left or right hip, as worn in most studies. ${ }^{[3]}$ In order to increase the probability of obtaining at least 3 consecutive days of pedometer data - as a minimum criterion for inclusion in the data analyses ${ }^{[1,15,16]}$ - a 5-consecutive-day protocol was decided as the number of days that participants were requested to wear the pedometer. Participants were asked to wear the pedometer throughout the day and to follow their usual routine of daily activities, and to remove the pedometer only when bathing or showering, swimming or sleeping. Participants were also informed that their daily results would be made available to them at the end of the study.

\section{Inclusion and exclusion criteria}

Employees attending the health screening event and/or willing to participate in the study were eligible for inclusion. Other inclusion criteria included being between the ages of 21 years (inclusive) and 50 years (exclusive) and willingness to wear a blinded pedometer during waking hours, for the duration of the study.

Employees were excluded for the following reasons: pregnancy; diagnosis or treatment of cancer; any other condition that could impact on physical activity; non-compliance to the pedometer wear; and participation in non-ambulatory physical activity (such as swimming and cycling) that might not be captured or be inaccurate through the pedometer reading.

\section{Ethical considerations and informed consent}

The study was approved by the Faculty of Health Sciences Human Research Ethics Committee (REC 348/2008) of the University of Cape Town. Permission was also obtained from corporate organisations to provide an onsite health screening event and to invite employees to participate in the study. Employees were provided with a participant information sheet detailing the purpose, objectives, procedures, requirements and potential risks of the study. They were thereafter required to sign an informed consent form.

\section{Measurements}

Anthropometric measurements included body height, waist circumference, body weight, body mass index and percentage body fat. Blood pressure (BP) and finger prick blood cholesterol and blood glucose were also measured.

\section{Self-perceived physical activity}

A questionnaire, administered as part of the health screening event, acquired information on physical activity habits. Questions relating to patterns of ambulatory physical activity enquired about the frequency (number of sessions of physical activity bouts), duration (approximate time spent in each bout) and intensity (estimated level of effort performed during each bout). This information was translated into two time-based categories, i.e. $<21$ minutes/day and $\geq 21$ minutes/day of aerobic activity, as an indicator of not meeting current guidelines and meeting current guidelines, respectively. These subgroups relate to current physical activity guidelines of 150 minutes of moderateintensity physical activity/week, ${ }^{[17]}$ approximated to 21 minutes/day.

\section{Pedometer data recording}

With particular reference to intensity-based steps, recently documented literature on intensity-based steps/day ${ }^{[18,19]}$ suggests a minimum of 100 steps/minute to be a reliable estimate of, and target for, moderateintensity physical activity (aerobic steps). Current literature also suggests that the accumulation of moderate-intensity physical activity in bouts of at least 10 minutes is acceptable in contributing towards meeting current physical activity guidelines. ${ }^{[17]}$

Using the graphical display of pedometer results, we considered bouts of $\geq 10$ consecutive minutes at a minimum average intensity of 100 steps/minute as moderate-intensity physical activity. The individual data were, as for the self-reported data, categorised into $<21$ minutes/day and $\geq 21$ minutes/day of aerobic activity as an indicator of not meeting and meeting current guidelines, respectively.

\section{Statistical analyses}

Both the self-reported data and the pedometer-measured data were grouped according to those meeting guidelines and those not meeting guidelines. Pedometer-determined steps/day were compared between these groups. A correlation analysis was performed to determine the extent of agreement between self-perceived and pedometer-measured physical activity. In this analysis, we compared those meeting current guidelines with those not meeting current guidelines in both pedometer-determined and self-reported data, respectively.

\section{Results}

In summary, 312 participants (147 men and 165 women; mean (standard deviation (SD)) 37 (9) years) were included in the analysis and completed the pedometer wear for a minimum of 3 consecutive days, with at least 10 hours/day of wear. The mean (SD) steps/day accumulated in men and women were 7476 (4 076) steps/day and 5769 (2 759) steps/day, respectively. A total 
of 112 participants (35.9\%) accumulated an average of $<5000$ steps/ day, typically classified as inactive. ${ }^{[18]}$ Only 41 participants (13.1\%) achieved an average of $\geq 10000$ steps/day, typically classified as moderately active. ${ }^{[18]}$ Within the moderately active group, most of the participants (73\%) also accumulated aerobic steps.

\section{Analysis of self-reported and pedometer data}

Fig. 1 presents the self-perceived and pedometer-determined mean steps/day data for men and women according to those meeting current guidelines and those not meeting guidelines.

Those participants who reported meeting guidelines $(n=63)$ accumulated significantly more steps/day than those not meeting guidelines (8 753 (4 251) steps/day v. 6022 (3 114) steps/day, respectively; $p<0.0001)$. A similar finding was observed for aerobic steps (1 772 (2 020) steps/day v. 421 (1 140) steps/day, respectively; $p<0.0001)$. Even after adjusting for age, gender and total steps/ day, significance was still noted in the number of aerobic steps/day between the two groups $(p<0.0001)$.

With regard to pedometer output, only 35 participants (11.2\%) of the total study group accumulated aerobic steps for an average of at least 21 minutes/day, and could therefore be classified as meeting current guidelines. These participants accumulated an average of 3951 (2 092) aerobic steps/day compared with 282 (603) aerobic steps/day in the $<21$ minute/day group, respectively; $p<0.0001$.
Even after adjusting for age, gender and total steps/day, significance was still noted in the difference between average aerobic steps/day accumulated in the two groups $(p<0.001)$.

Participants categorised into the $\geq 21$ minute/day group also accumulated significantly more total steps/day than those not meeting guidelines (10 092 (3 445) steps/day v. 6129 (3 302) steps/day, respectively; $p<0.0001)$.

\section{Association between pedometer-determined and self-reported} ambulatory physical activity

A correlation between self-reported and pedometer-measured ambulatory physical activity, using the meeting guidelines and not meeting guidelines categories, indicated that approximately $80 \%$ $(n=249)$ of the total group did not meet guidelines, according to self-reported data. The pedometer analysis showed that nearly $90 \%$ $(n=277)$ did not meet guidelines.

Of the 63 participants $(20.2 \%$; $N=312)$ who met guidelines (selfreported), only $27 \%(n=17)$ met guidelines according to pedometer data. Thirty-five participants were classified as meeting guidelines (pedometer determined), but more than half of these participants (51.4\%) did not meet guidelines according to the self-reported data.

Participants who reported meeting guidelines were, however, more likely to meet guidelines (pedometer measured) (40\%) than those who reported not meeting guidelines $(8 \%)$.

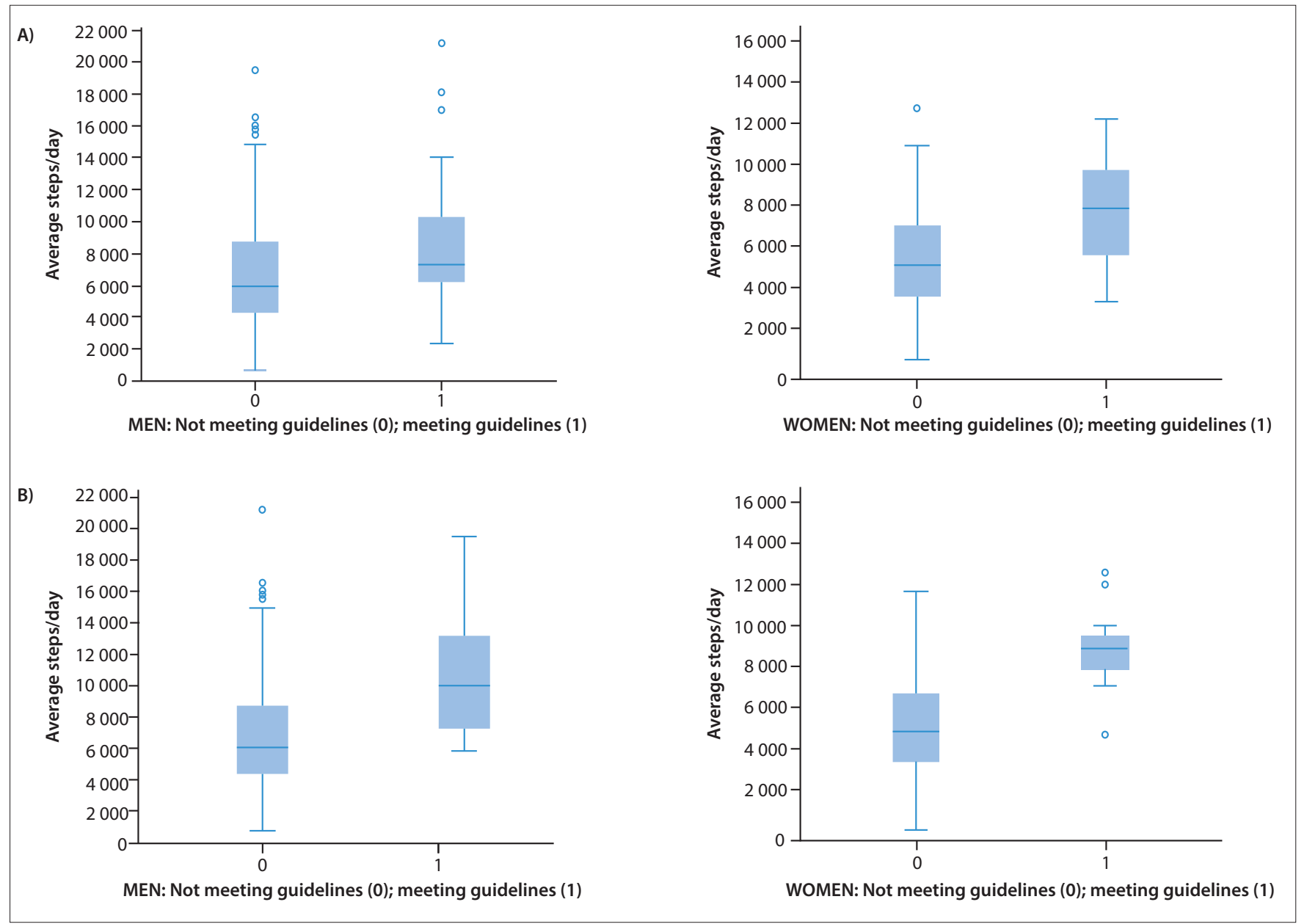

Fig. 1. Steps per day in relation to physical activity guidelines: (A) self-reported; (B) pedometer determined. 


\section{Discussion}

Our findings showed that those participants who met guidelines (selfreported) accumulated an average of 2731 more steps/day than those who did not meet guidelines $(p<0.001)$. This difference was more marked in women (2 781 steps/day; $p<0.001$ ) than men (2 247 steps/ day; $p<0.005)$. In a study by Miller and Brown ${ }^{[6]}$ in 2004, a comparable outcome was noted. Participants $(N=185)$ who met guidelines took an average of 1357 steps/day more than those who did not meet guidelines. Similarly, the difference was more marked among women (1 684 steps/day) than men (1 019 steps/day). ${ }^{[6]}$

A popular public health message relating to pedometry is the 10000 steps/day concept, which shows positive health outcomes in those achieving this target compared with those not achieving 10000 steps/day, ${ }^{[6,11-13]}$ while a steps/day value of $<5000$ steps/day has generally been classified as inactive. ${ }^{[18]}$ Within the context of the 10000 steps/day guideline, Miller and Brown ${ }^{[6]}$ demonstrated that the accumulation of 10000 steps/day did not always correlate with meeting guidelines. The study reported that nearly $40 \%$ of participants who met guidelines did not reach the 10000 steps/ day target. The study also reported that $\sim 10 \%$ of participants who did achieve the 10000 steps/day target did not meet guidelines. ${ }^{[6]}$ This implies that a large proportion of people meeting current guidelines do not reach 10000 steps/day, yet most people reaching 10000 steps/day meet current guidelines.

Our study has shown a more exaggerated finding to that in Miller and Brown ${ }^{[6]}$ in that $71.4 \%$ of participants who met guidelines $(n=63)$ (self-reported), did not achieve the 10000 steps/day target.

With regard to those achieving 10000 steps/day, our findings show a somewhat different outcome to that of Miller and Brown, ${ }^{[6]}$ as $56.1 \%$ of participants who reached the 10000 steps/day target ( $n=41)$ still did not meet physical activity guidelines (self-reported). Only $37.1 \%$ of participants who met guidelines ( $n=35)$ according to pedometer data achieved the 10000 steps/day target. Consequently, $68.3 \%$ of participants who did not achieve the 10000 steps/day target $(n=41)$ did not meet guidelines (self-reported). A large proportion of those individuals achieving 10000 steps/day were also shown not to accumulate sufficient steps at a moderate intensity.

Recent literature exemplifies that even in the absence of meeting the 10000 steps/day recommendation, the impact of intensity-based steps (moderate-intensity physical activity) is significantly greater than volume alone. ${ }^{[12]}$ The implication of this, in keeping with our findings, emphasises the emerging importance of intensity-based steps. As such, the need for public health messages that emphasise the importance of both intensity and volume of steps/day is particularly pertinent.

\section{Study strengths}

This research is among the first pedometer-based studies conducted in South Africa, within an urban context, that establishes the association between pedometer-measured ambulatory physical activity and selfreported information.

Our study has more specifically related self-reported physical activity and pedometer-determined steps/day, with consideration of both volume and intensity of daily steps accumulated, and current guidelines. The presentation of our results within this context provides a novel application to pedometer-based research that can be applied in similar future studies.
The intensity-based output (from pedometer data) additionally included steps accumulated over a minimum duration and intensity. This allowed us to determine step/minute rates, so as to closely relate to moderate-intensity physical activity and current guidelines. ${ }^{[17-19]}$ This application is also a novel application in pedometer-based research and provides an opportunity for further application and modification.

Additionaly, we provide some estimate of the level of agreement between self-reported and pedometer-determined physical activity.

\section{Study limitations}

Pedometers measure ambulatory physical activity. Our comparison was, therefore, limited to participants performing activities more specific to ambulation and did not include activities such as swimming, cycling and weight training.

The 100 steps/minute criterion, used as a baseline criterion for moderate-intensity physical activity, may not always be a true indication of moderate-intensity physical activity at an individual level. For example, this estimate may likely be affected by factors such as aerobic fitness and heart rate response to physical activity. A number of studies that have directly measured moderate intensity as three metabolic equivalents (3 METs) have, however, concluded that 100 steps/minute is a reasonable heuristic value indicative of moderate-intensity physical activity. ${ }^{[13,18]}$

The subgrouping of data according to intensity-based categories, using 21 minutes/day of aerobic activity as a proxy for current physical activity recommendations, may be viewed as a further limitation. However, this categorisation has provided some level of differentiation of ambulatory physical activity according to intensity and duration of steps/day. This has allowed us to relate volume and intensity-based pedometer data to self-reported data, with particular reference to current guidelines, as a unique application to pedometry.

Pedometers are typically used to measure volume of steps/day. The reliability of information on intensity-based steps and the refinement of intensity-based steps through pedometry, and the application thereof to current guidelines may raise concern. This does, however, provide some level of determining a more direct measure of intensitybased ambulatory physical activity than self-reported means, and is particularly valuable as a less costly alternative to other direct measures.

\section{Conclusion}

Our study highlights the association between self-reported physical activity patterns and objectively measured ambulatory physical activity, with particular reference to both volume and intensity of steps/day, and current guidelines.

In view of our results showing a very small percentage of participants meeting current guidelines (from both pedometer data and self-reported data), further studies using a similar but broader approach could enhance the reliability of our findings.

The importance of intensity-defined steps/day recommendations is highlighted. The application of such recommendations in pedometerbased interventions may provide useful insights on its effects in improving fitness and health.

Acknowledgements. We acknowledge the following organisations for funding this project: Durban University of Technology (DUT) and the National Research Foundation (Thuthuka). 


\section{References}

1. Prince SA, Adamo KB, Hamel ME, Hardt J, Gorber SC, Tremblay MS. A comparison of direct versus self-report measures for assessing physical activity in adults: A systematic review. Int J Behav Nutri Phys Act 2008;5(1):56. [http://dx.doi.org/10.1186/14795868-5-56]

2. Dishman RK, Washburn RA, Schoeller DA. Measurement of physical activity. Quest 2001;53(3):295-309. [http://dx.doi.org/10.1080/00336297.2001.10491746]

3. Tudor-Locke C. Taking Steps Toward Increased Physical Activity: Using Pedometers to Measure and Motivate. Washington DC: President's Council on Physical Fitness and Sports, 2002.

4. Ainslie PN, Reilly T, Westerterp KR. Estimating human energy expenditure: A review of techniques with particular references to doubly labelled water. Sports Med 2003;33(9):683-698.

5. Shephard RJ. Limits to the measurement of habitual physical activity by questionnaires. Br J Sports Med 2003;37(3):197-206. [http://dx.doi.org/10.1136/ bjsm.37.3.197]

6. Miller R, Brown W. Meeting physical activity guidelines and average daily steps in a working population. J Phys Act Health 2004;1(3):218-226.

7. Strycker LA, Duncan SC, Chaumeton NR, Duncan TE, Toobert DJ. Reliability of pedometer data in samples of youth and older women. Int J Behav Nutr Phys Act 2007;4:4-11. [http://dx.doi.org/10.1186/1479-5868-4-4]

8. Tudor-Locke C, Ainsworth BE, Thompson RW, Matthews CE. Comparison of pedometer and accelerometer measures of free-living physical activity. Med Sci Sports Exerc 2002;34(12):2045-2051. [http://dx.doi.org/10.1249/01. MSS.0000039300.76400.16]

9. Matthews CE, Chen KY, Freedson PS, et al. Amount of time spent in sedentary behaviors in the United States, 2003-2004. Am J Epidemiol 2008;167(7):875-881. [http://dx.doi.org/10.1093/aje/kwm390]
10. Matton L, Wijndaele K, Duvigneaud N, Duquet W, Philippaerts R, Thomis M. Reliability and validity of the Flemish Physical Activity Computerized Questionnaire in adults. Res Q Exerc Sport 2007;78(4):293-306. [http://dx.doi.org/10.1080/02701367.2007.10599427]

11. Tudor-Locke C, Bassett DRJ. How many steps/day are enough? Preliminary pedometer indices for public health. Sports Med 2004;34(1):1-8. [http://dx.doi. org/10.2165/00007256-200434010-00001]

12. Wilde BE, Sidman CL, Corbin CB. A 10000 step count as a physical activity target for sedentary women. Res Q Exerc Sport 2001;72(4):411-414. [http://dx.doi.org/10.1 080/02701367.2001.10608977]

13. Tudor-Locke C, Craig CL, Brown WJ, et al. How many steps/day are enough? For adults. Int J Behav Nutr Phys Act 2011;8:79-97. [http://dx.doi.org/10.1186/14795868-8-79]

14. Incorporated Omron Health. Instruction manual: Pocket pedometer-model HJ720ITC2007. The Netherlands: Incorporated Omron Health, 2007.

15. Rowe DA, Kemble CD, Robinson TS, Mahar MT. Daily walking in older adults: Dayto-day variability and criterion-referenced validity of total daily step counts. J Phys Act Health 2007;4(4):434-446.

16. Hart TL, Swartz AM, Cashin SE, Strath SJ. How many days of monitoring predict physical activity and sedentary behaviour in older adults? Int J Behav Nutr Phys Act 2011;8:62. [http://dx.doi.org/10.1186/1479-5868-8-62]

17. World Health Organization. Global Recommendations on Physical Activity for Health. Geneva: World Health Organization, 2010.

18. Tudor-Locke C, Sisson SB, Collova T, Lee SM, Swan PD. Pedometer-determined step count guidelines for classifying walking intensity in a young ostensibly healthy population. Can J Appl Physiol 2005;30(6):666-676.

19. Marshall SJ, Levy SS, Tudor-Locke CE, et al. Translating physical activity recommendations into a pedometer-based step goal: 3000 steps in 30 minutes. Am J Prev Med 2009;36(5):410-415. [http://dx.doi.org/10.1016/j.amepre.2009.01.021] 